\title{
The Postsynaptic Glutamate Receptor Subunit DGluR-IIA Mediates Long-Term Plasticity in Drosophila
}

\author{
Stephan J. Sigrist, ${ }^{*}$ Philippe R. Thiel, ${ }^{*}$ Dierk F. Reiff, and Christoph M. Schuster \\ Friedrich-Miescher-Laboratorium der Max-Planck-Gesellschaft, 72076 Tübingen, Germany
}

The developing neuromuscular junctions (NMJs) of Drosophila larvae can undergo long-term strengthening of signal transmission, a process that has been shown recently to involve local subsynaptic protein synthesis and that is associated with an elevated synaptic accumulation of the postsynaptic glutamate receptor subunit DGluR-IIA. To analyze the role of altered postsynaptic glutamate receptor expression during this form of genetically induced junctional plasticity, we manipulated the expression levels of two so far-described postsynaptic receptor subunit genes, dglur-IIA and dglur-IIB, in wild-type animals and plasticity mutants. Here we show that elevated synaptic expression of DGluR-IIA, which was achieved by direct transgenic overexpression, by genetically increased subsynaptic protein synthesis, or by a reduced dglur-IIB gene copy number, results in an increased recruitment of active zones, a corresponding enhancement in the strength of junctional signal transmission, and a correlated addition of boutons to the NMJ. Ultrastructural evidence demonstrates that active zones appear throughout

The localized synthesis of proteins at synapses represents an attractive mechanism to mediate site-specific and long-lasting modifications of neuronal function and connectivity. This form of rapid and localized gene expression at synapses has been widely implicated in plasticity phenomena (Martin et al., 2000), including neurotrophin-induced long-term potentiation (Kang and Schuman, 1996), synapse-specific tagging during long-term plasticity (Frey and Morris, 1997; Martin et al., 1997; Casadio et al., 1999), and metabotropic glutamate receptor (GluR)-dependent long-term depression (Linden, 1996; Huber et al., 2000; Manahan-Vaughan et al., 2000). It therefore appears that longlasting changes in neuronal communication are to a large extent controlled by the local regulation of synaptic protein synthesis. However, the processes that mediate such alterations downstream of synaptic translation are less well described.

Localized subsynaptic protein synthesis has been shown re-

Received April 17, 2002; revised June 10, 2002; accepted June 11, 2002.

This work was supported by the Max-Planck-Society. We thank A. DiAntonio (Washington University, St. Louis, MO), C. S. Goodman (University of California, Berkeley, CA), Y. Kidokoro (Gunma University, Gunma, Japan), and P. Lasko for generous gifts of reagents. We also thank E. M. Illgen, M. Langegger, and C. Strohm for excellent technical assistance, H. Schwarz for advice during ultrastructural studies, and W. Hoch for critical comments on this manuscript and helpful discussions.

*S.J.S. and P.R.T. contributed equally to this work.

Correspondence should be addressed to Christoph M. Schuster, FriedrichMiescher-Laboratorium der Max-Planck-Gesellschaft, Spemannstrasse 39, 72076 Tübingen, Germany. E-mail: Christoph.Schuster@tuebingen.mpg.de.

S. J. Sigrist's present address: European Neuroscience Institute Göttingen, MaxPlanck-Institute for Biophysical Chemistry, Waldweg 33, 37073 Göttingen, Germany.

Copyright (C) 2002 Society for Neuroscience $\quad 0270-6474 / 02 / 227362-11 \$ 15.00 / 0$
NMJs at a typical density regardless of genotype, suggesting that the space requirements of active zones are responsible for the homogeneous synapse distribution and that this regulation results in the observed growth of additional boutons at strengthened NMJs. These phenotypes were suppressed by reduced or eliminated DGluR-IIA expression, which resulted from either a reduced dglur-IIA gene copy number or transgenic overexpression of DGluR-IIB. Our results demonstrate that persistent alterations of neuronal activity and subsynaptic translation result in an elevated synaptic accumulation of DGluR-IIA, which mediates the observed functional strengthening and morphological growth apparently through the recruitment of additional active zones.

Key words: synaptic translation; glutamate receptor; subunit composition; active zones; T-bars; long-term strengthening; synapse recruitment; morphological plasticity; consolidation; Fasciclin II; neuromuscular junction; Drosophila

cently to be involved in the persistent strengthening of larval neuromuscular junctions (NMJs) of Drosophila (Sigrist et al., 2000), which can be observed in mutants with chronically increased neuronal activity ( $\mathrm{eg}^{1}, S h^{102}$ ) and elevated cAMP levels $\left(d n c^{M 14}\right)$ (Budnik et al., 1990). Signal transmission at these NMJs is primarily conducted at glutamatergic synapses, which are localized on larval body wall muscles and which express at least two ionotropic glutamate receptor subunits, DGluR-IIA (Schuster et al., 1991) and DGluR-IIB (Petersen et al., 1997). On the basis of electrophysiological examinations of mutants and overexpressed transgenes of either glutamate receptor subunit gene, it has been shown that both subunits affect the synaptic physiology in an opposing manner (Petersen et al., 1997; DiAntonio et al., 1999), suggesting that the subunit composition of postsynaptic glutamate receptors represents an important regulatory parameter for the strength of signal transmission at larval NMJs. Importantly, we have found recently that mRNAs encoding the postsynaptic glutamate receptor subunit DGluR-IIA are stored within the subsynaptic reticulum of NMJs and that a genetic elevation of subsynaptic protein synthesis is associated with an increased synaptic accumulation of DGluR-IIA and a persistently enhanced signal transmission (Sigrist et al., 2000). These findings provided evidence that the postsynaptic receptor subunit composition can be dynamically regulated by subsynaptic translation, and they suggest that such regulation may play an important role during the development of Drosophila NMJs.

This study was aimed to better understand the role of altered DGluR-IIA expression during genetically induced forms of plasticity at Drosophila NMJs. We manipulated the expression of both 
postsynaptic glutamate receptor subunits and assessed the physiological, morphological, and ultrastructural consequences in wild-type animals and in model genotypes of activity-dependent junctional plasticity. We provide evidence that increased postsynaptic DGluR-IIA expression alone is sufficient and necessary to recruit additional active zones, which result in the persistent strengthening of junctional signal transmission. The perisynaptic space requirement of these additional active zones can explain why such persistently strengthened NMJs develop more boutons. We further show that an increased synaptic DGluR-IIA accumulation can be suppressed by DGluR-IIB expression, providing a potential alternative mechanism to tune the DGluR-IIA levels at synapses during development. Thus, it appears that the effects of subsynaptic protein synthesis are mediated by the described DGluR-IIA-dependent mechanisms.

\section{MATERIALS AND METHODS}

Immunostaining and electrophysiology were performed as described by Sigrist et al. (2000).

Larval culture. All larvae were raised under tightly controlled standardized culture conditions (constant $25^{\circ} \mathrm{C}, 65 \%$ humidity, and high animal density) and used as midthird instar larvae shortly before the onset of the wandering stage.

Immunofluorescence quantification. Junctional immunoreactivity levels of DGluR-IIA were quantified in double-labeled larval preparations with the invariant anti-HRP immunoreactivity at NMJs as an internal staining standard. Five to nine type Ib boutons (muscle 6/7, abdominal segment A2) were selected in the anti-HRP channel of a recorded confocal image stack, and the average fluorescence signal of this selection was determined for both channels. The signal ratios of DGluR-IIA/HRP of at least two nonoverlapping areas per NMJ were accumulated from 6-20 animals per genotype. All obtained data were expressed relative to those of the respective control genotypes, which were set to $100 \%$.

Quantification of junction size and subsynaptic translation aggregates. Larval fillet preparations of various genotypes were simultaneously stained with antibodies recognizing the translation initiation factor eIF4E (a generous gift from Paul Lasko, McGill University, Montreal, Québec, Canada) and the cell adhesion molecule Fasciclin II (FasII, monoclonal antibody 1D4; a generous gift from Corey Goodman, University of California, Berkeley, CA). The number of synaptic boutons per NMJ (muscle 6/7, abdominal segment A2) was determined in the FasII immunofluorescence channel at the microscope. Previous experiments have revealed that bouton quantifications using FasII-labeled NMJs were similarly reliable as those performed with an antibody recognizing the presynaptic vesicle protein synaptotagmin. In addition, the measured muscle surface area was used as a fine-scale developmental criterion to normalize the obtained bouton counts (number of boutons divided by the muscle surface area; Sigrist et al., 2000). All quantifications were performed double-blind.

Subsynaptic translation aggregates were scored in the eIF4E immunofluorescence channel by counting the number of boutons (FasII channel) per NMJ (muscle 6/7, abdominal segments A2-A5) that showed strong eIF4E immunoreactivity. This method of quantification is likely to underscore the actual junctional eIF4E immunoreactivity, which besides the large aggregates occasionally showed morphologically less confined weaker signals.

Electron microscopy. Larvae were filleted and processed for ultrastructural analysis as described previously (70 min fixation in ice-cold $4 \%$ paraformaldehyde and PBS) (Schneider et al., 2000). Serial ultrathin sections of boutons (muscle 6/7, segment A2, 4-10 branches from two animals per genotype; $>2300$ sections in total) were photographed at 21,000-fold enlargement with a CM10 electron microscope (Philips, Endhoven, The Netherlands), scanned, and reconstructed using NIH Image 1.62. We determined the surface area by measuring the bouton perimeters of every section and integrating them over the depth of the individual reconstruction ( $87 \mathrm{~nm}$ thickness per section). Perimeters of missing sections (average, 46\%) were extrapolated from neighboring sections. The sizes of individual synapses (dense areas) were similarly determined by integrating the lengths of the electron-dense areas per section throughout their appearance in the following sections. The presence or absence of presynaptic dense bodies (T-bars) was scored. From these raw data, we derived the number and density of synapses of each reconstructed branch (number of dense areas/surface area of that branch). For representation of these ultrastructural data as accurately as possible, they were weighted according to the quality of representation per branch, which was given by the percentage of ultrathin sections that could be analyzed from a reconstructed branch [(length of reconstructed branch $\times$ percentage of sections)/sum of all weighting factors of that genotype]. These weighing factors were then used to calculate mean values \pm SEM from a total of 4-10 branches per genotype (Table 1).

Genetics. All genetic elements used in this study have been described previously (Petersen et al., 1997; DiAntonio et al., 1999; Sigrist et al., 2000). dglur-II $A^{A D 9}$ eliminates dglur-IIA.df(2L)clh4 and dglur-IIA $A^{A 22}$ are genetically independent and remove both the dglur-IIA and dglur-IIB locus. pabp ${ }^{P 970}$ is a loss-of-function allele of the pabp locus. Transgenes were $\mathrm{P}\left(\mathrm{UAS}-\text { gdglur-IIA; } \mathrm{w}^{+}\right)^{g 10}, \mathrm{P}\left(\mathrm{UAS}-\right.$ gdglur-IIB; $\left.\mathrm{w}^{+}\right)$, P(UAS-gdglur$\left.I I A ; \mathrm{w}^{+}\right)^{g 9}, \mathrm{P}\left(\mathrm{UAS}_{-}\right.$-fasII; $\left.\mathrm{w}^{+}\right)$, and $\mathrm{P}\left(\mathrm{UAS}-\right.$ pabp $\left.; \mathrm{w}^{+}\right) . \mathrm{P}\left(\mathrm{Mhc}-\mathrm{Gal} 4 \mathrm{ry}^{+}\right)$ and $\mathrm{P}\left(24 \mathrm{~B}-\mathrm{Gal} ; \mathrm{w}^{+}\right)$are muscle-specific Gal4-driver lines. $\mathrm{P}(\mathrm{E} 62-\mathrm{Gal}$; $\mathrm{w}^{+}$) is Gal4 expression in MN3a (Schuster et al., 1996a).

\section{RESULTS}

\section{Overexpression of DGluR-IIA results in an increased number of DGluR-IIA-positive synapses}

The glutamatergic synapses of larval NMJs can be identified on the surface of junctional boutons as small patches that are immunopositive with antibodies recognizing the glutamate receptor subunit DGluR-IIA (Fig. 1) (Saitoe et al., 1997; Sone et al., 2000; Sigrist et al., 2000; Parnas et al., 2001) and epitope-tagged fusion proteins of DGluR-IIA and DGluR-IIB (Petersen et al., 1997; DiAntonio et al., 1999; Parnas et al., 2001). The position of synapses can also be estimated through the perisynaptic expression of the cell adhesion molecule Fasciclin II, which shows weak or no immunoreactivity within synaptic zones (Fig. 1A, arrows) (Sone et al., 2000). To assess the functional consequences of dynamically altered expression of glutamate receptor subunits, we first examined the synaptic expression profile of DGluR-IIA subunits in wild-type animals. We found that DGluR-IIA is expressed in typical patches within NMJs in only a subset of synapses (Fig. $1 A$, arrowheads), whereas most synaptic zones showed weak or no detectable DGluR-IIA-specific immunoreactivity (Fig. $1 A$, arrows). A similar analysis of the synaptic DGluRIIB expression was prevented by the current lack of appropriate antibodies to specifically detect endogenous DGluR-IIB subunits. Thus, larval NMJs harbor a heterogeneous set of synapses that differ in the composition of postsynaptic glutamate receptor subunits.

To genetically simulate the elevated DGluR-IIA levels seen in previously described mutants with strengthened signal transmission (Sigrist et al., 2000), we overexpressed this glutamate receptor subunit by using a genomic dglur-IIA transgene (UAS-gdglurII $A$; Petersen et al., 1997; DiAntonio et al., 1999) in combination with the muscle-specific driver line Mhc-Gal4. This resulted in a significant elevation of the junctional DGluR-IIA immunoreactivity (Fig. $1 C$ ) that was primarily attributable to an increase in the number of DGluR-IIA positive synapses (Fig. $1 B$, red chan$n e l)$. We compared this DGluR-IIA phenotype with that seen in animals heterozygous for a loss-of-function allele of pabp (Fig. $\left.1 C, p_{a b p} p^{970} /+\right)$, a gene encoding the translation-initiation factor poly(A)-binding protein (PABP) (Gallie, 1998). We have shown recently (Sigrist et al., 2000) that this genotype results in an increased occurrence of subsynaptic eIF4E aggregates (see Fig. 6) and in an elevated synaptic accumulation of DGluR-IIA. Both genotypes showed similarly increased synaptic immunoreactivity (Fig. 1C) and distribution of DGluR-IIA. The transgenic overexpression of DGluR-IIA also resembled to a large extent the increased synaptic accumulation of DGluR-IIA seen in several other genotypes, which are considered to represent genetic mod- 

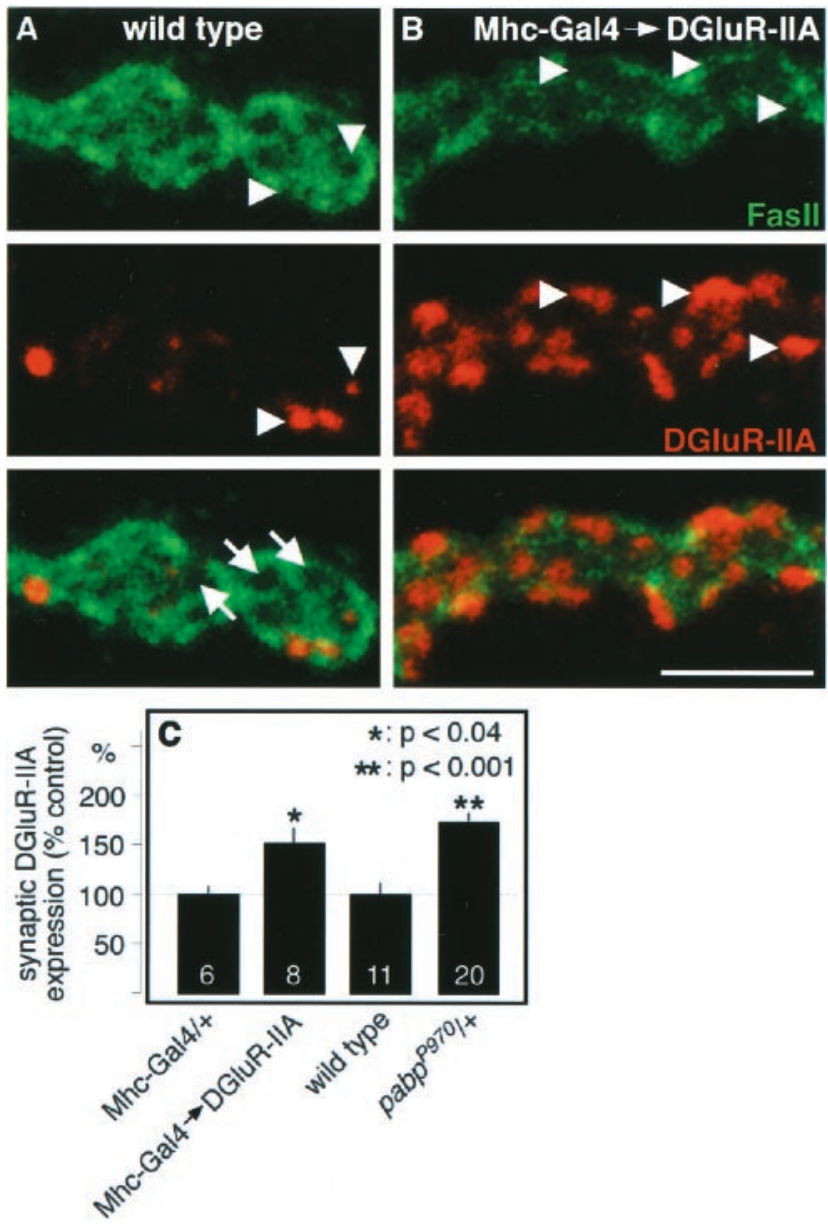

Figure 1. Transgenic overexpression of DGluR-IIA in larval muscles increases the number of DGluR-IIA-positive synapses at NMJs. $A$, At NMJs of wild-type larvae, the cell adhesion molecule FasII (green channel) is localized to perisynaptic areas of junctional boutons, leaving free disk-shaped zones, which harbor glutamatergic synapses (Sone et al., 2000). The glutamate receptor subunit DGluR-IIA (red channel) is detected in a subset of synapses (arrowheads), whereas other synaptic areas show weak or no detectable DGluR-IIA immunoreactivity (arrow). $B$, Transgenic overexpression of DGluR-IIA $(M h c-G a l 4 \rightarrow D G l u R-I I A)$ results in an increased junctional DGluR-IIA immunoreactivity that is largely attributable to an increased number of DGluR-IIA-positive synapses. Note the visibly reduced FasII immunoreactivity in this genotype. Scale bar, $5 \mu \mathrm{m}$. $C$, Transgenic DGluR-IIA overexpression results in a significant increase in total junctional DGluR-IIA immunoreactivity (see Materials and Methods), which is similar to that seen in larvae with elevated subsynaptic translation ( $p a b p^{P 970} /+$; Sigrist et al., 2000). Data represent means $\pm \mathrm{SEM}$.

els of activity-dependent strengthening at larval NMJs $\left(d n c^{M 14}\right.$, pabp $^{E P 0310} / d f$, Mhc-Gal4->PABP, and Mhc-Gal4->eIF4e; Sigrist et al., 2000).

\section{Elevated DGluR-IIA expression is sufficient and necessary to mediate translation-induced strengthening of signal transmission at larval NMJs}

Genetic manipulations of the expression levels of DGluR-IIA and DGluR-IIB have already shown that the subunit composition of glutamate receptors affects the postsynaptic sensitivity to released glutamate. For example, strong transgenic overexpression of DGluR-IIA resulted in a dose-dependent increase in quantal size (Petersen et al., 1997), whereas similar overexpression of DGluR-IIB showed reduced postsynaptic sensitivity to the trans- mitter (DiAntonio et al., 1999). Additional experiments, in which the lethality of the double knock-out of dglur-IIA and dglur-IIB genes was rescued by the transgenic expression of DGluR-IIA or DGluR-IIB, showed that the single-channel properties of DGluR-IIA-rescued synapses resembled those of wild-type synapses even in the absence of DGluR-IIB expression. In contrast, synapses composed of DGluR-IIB without DGluR-IIA showed a strongly reduced time constant of desensitization and significantly smaller quantal sizes (DiAntonio et al., 1999). From these and additional data, which showed that quantal sizes were significantly smaller when DGluR-IIA subunits were phosphorylated by PKA (Davis et al., 1998), it emerged that DGluR-IIA represents the primary glutamate receptor subunit that is required for normal postsynaptic function and sensitivity. Compared with this prominent role of DGluR-IIA, the subunit DGluR-IIB appeared to play a rather modulatory role.

Given that $p a b p^{P 970} /+$ animals showed an increased synaptic accumulation of DGluR-IIA (Fig. 1C) and strengthened junctional signal transmission (Sigrist et al., 2000), we then tested whether this strengthening relies on the dynamic upregulation of DGluR-IIA expression. For this we recorded evoked and miniature excitatory junctional currents (eEJCs and mEJCs, respectively) from muscle 6 in abdominal segment A2 of third instar larvae. From these measurements we derived the junctional quantal content (mean eEJCs/mean mEJCs) as an estimate of the number of vesicles released per stimulus. Consistent with our previous observations (Sigrist et al., 2000), NMJs of $p a b p^{P 970} /+$ animals showed unaltered postsynaptic sensitivity compared with wild-type NMJs (Fig. 2B, mEJCs) but significantly larger evoked responses (Fig. 2B, eEJCs). The junctional quantal content was therefore significantly larger at $p a b p^{P 970} /+$ NMJs compared with wild type (Fig. $2 C$, black bars) and was associated with an elevated frequency of spontaneous vesicle fusion events (Fig. 2C, white bars). Intriguingly, we observed similar physiological phenotypes (unaltered quantal sizes, enhanced evoked release of presynaptic vesicles, and a significantly elevated frequency of mEJCs) at NMJs that transgenically overexpressed DGluR-IIA (Fig. 2, MhcGal4 $\rightarrow$ DGluR-IIA), demonstrating that mild overexpression of DGluR-IIA alone is sufficient to phenocopy the physiological effects of genetically elevated subsynaptic translation. Furthermore, the elimination of one dglur-IIA gene copy in $p a b p^{P 970} /+$ animals resulted in an almost complete suppression of enhanced junctional signal transmission (Fig. $2 C$, pabp ${ }^{P 970}$, dglur-II $A^{A D 9}$ + ), indicating that animals with genetically restricted DGluR-IIA expression are incapable of developing a strengthened NMJ. These experiments therefore establish that elevated DGluR-IIA accumulation at developing NMJs is sufficient and necessary to strengthen junctional signal transmission in $\mathrm{pabp}^{P 970} /+$ animals. Thus, translation-induced strengthening of developing larval NMJs appears to be mediated by an elevation in the synaptic expression of DGluR-IIA.

How could an increased DGluR-IIA expression result in the observed strengthening of junctional signal transmission? A clue toward an answer came from the fact that the apparent number of DGluR-IIA-harboring synapses is increased at all so far analyzed NMJs that showed elevated quantal contents (Fig. 1) (Sigrist et al., 2000). In addition, these NMJs shared a considerable elevation of the frequency of mEJCs (Fig. $2 C$, white bars). Both observations are consistent with the idea that these NMJs harbor an increased number of active synapses. In fact, we will later in this study provide ultrastructural evidence (see Fig. 6) that the total number of active zones per NMJ is indeed significantly 


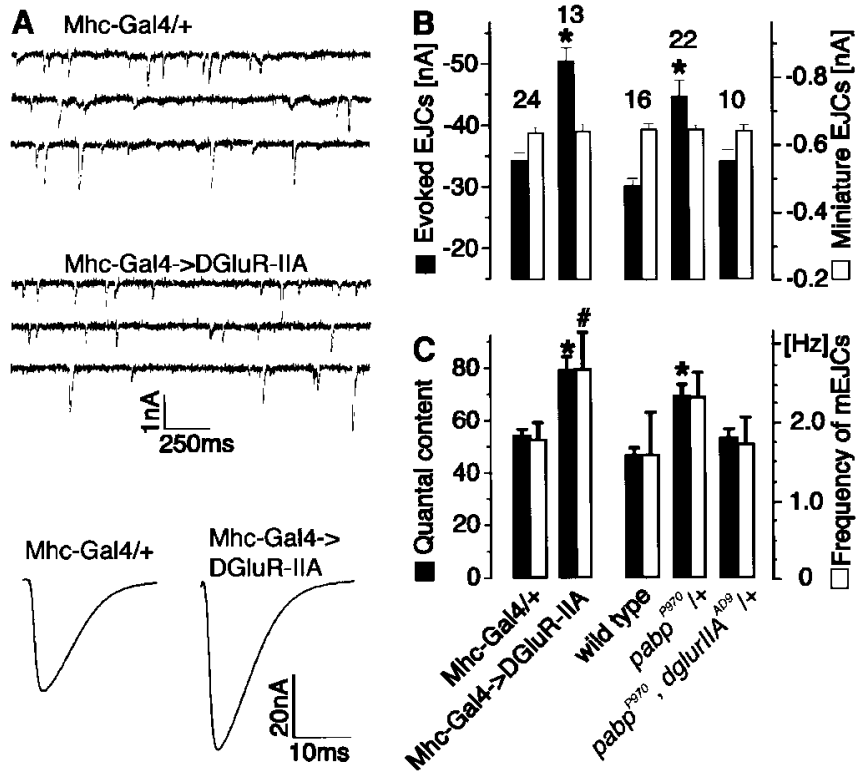

Figure 2. Increased DGluR-IIA expression is required for the long-term strengthening of signal transmission at larval NMJs. A, Two-electrode voltage-clamp recordings of eEJCs and mEJCs from muscle 6 of abdominal segment A2. Representative traces of mEJC recordings (top panels) and average traces of 10 consecutively recorded eEJCs of the indicated genotypes are shown. $B$, The mean amplitudes of mEJCs are indistinguishable among all analyzed genotypes (white bars). Compared with controls, the eEJCs are significantly larger in animals overexpressing DGluR-IIA either by transgenic means $(M h c-G a l 4 \rightarrow D G l u R-I I A)$ or by sensitized subsynaptic translation $\left(\right.$ pabp $\left.^{P 970} /+; p \ll 0.001\right)$. This amplitude increase of eEJCs is efficiently suppressed by the removal of one copy of the dglur-IIA gene ( $p a b p^{P 970}$, dglur-IIIAAD9/+). C, The derived junctional quantal content (mean amplitude eEJCs/mean amplitude of mEJCs) and thus the efficacy of signal transmission show similarly significant changes in the analyzed genotypes compared with controls (black bars). Enhanced signal transmission is paralleled by an elevated frequency of mEJCs (white bars; $p<0.02$; sampled over $2 \mathrm{~min}$ ). Data in $B$ and $C$ are derived from the indicated number of cells (number above bars in $B$ ) and represent means \pm SEM.

increased in such animals. We therefore conclude that elevated DGluR-IIA expression is required for an increased functional recruitment of active synapses that in turn can give rise to the observed strengthening of junctional signal transmission.

\section{DGluR-IIA mediates a coordinated functional and morphological growth of NMJs}

Accumulating evidence from several recent studies showed that the strength of junctional signal transmission is strictly coupled to the morphological development of NMJs (Cheung et al., 1999; Sigrist et al., 2000; Sanyal et al., 2002), as long as the excitability of the postsynapse itself and the postsynaptic cell or the growth mechanisms themselves are not disturbed (Schuster et al., 1996; Petersen et al., 1997; Featherstone et al., 2000; Wan et al., 2000; DiAntonio et al., 2001; Paradis et al., 2001; Aberle et al., 2002). For example, genetic manipulations of the expression level of the cell adhesion molecule Fasciclin II did result in significant changes of presynaptic bouton numbers; however, these morphological changes did not cause a large-scale alteration in the strength of junctional signal transmission (Schuster et al., 1996; Stewart et al., 1996). In contrast, animals with genetically elevated subsynaptic protein synthesis developed NMJs with unaltered mEJCs but larger eEJC amplitudes and proportionally more synaptic boutons compared with controls (Sigrist et al., 2000).
These observations suggest that such enhanced junctional signal transmission can trigger the addition of presynaptic boutons. Consistent with this idea, we found that animals that chronically overexpressed DGluR-IIA driven by either of two muscle-specific Gal4-driver lines (24B-Gal4 and Mhc-Gal4) showed enlarged NMJs compared with their respective controls (Mhc-Gal4/+ and 24B-Gal4/+) or wild-type animals (Fig. 3A,B). The larger size of NMJs was evident in a significant increase in the number of synaptic boutons (Fig. $3 C$ ), which were typical in shape and size (Fig. $3 B$ ). In addition, the number of boutons and the strength of evoked signal transmission of DGluR-IIA-overexpressing NMJs (Mhc-Gal4 $\rightarrow$ DGluR-IIA) showed a similar relationship as seen in animals with elevated subsynaptic translation (Fig. $3 E$, pabp $p^{P 97 /+)}$. Importantly, the removal of one $\operatorname{dglur}$-IIA gene copy in $p a b p^{P 970} /+$ animals resulted in a suppressed strength of junctional transmission (Fig. 2) and in a corresponding suppression of junctional growth (Figs $3 E, 4 E$ ). These data demonstrate that the elevated expression of DGluR-IIA is sufficient and necessary to induce a correlated functional and morphological strengthening of NMJs.

\section{DGluR-IIB expression negatively affects the synaptic accumulation of DGluR-IIA}

In contrast to DGluR-IIA, the transgenic overexpression of the glutamate receptor subunit DGluR-IIB did not result in significant alterations of the morphological development of larval NMJs compared with controls (Fig. 3D) (Mhc-Gal4/+ vs Mhc$\mathrm{Gal} \rightarrow$ DGluR-IIB, $p \gg 0.1$; 24B-Gal4/+ vs $24 \mathrm{~B}-$ Gal4 $\rightarrow$ DGluRIIB, $p>0.1$ ). In addition, the simultaneous overexpression of both glutamate receptor subunits, DGluR-IIA and DGluR-IIB, showed a significant suppression of the bouton addition seen in Mhc-Gal4 $\rightarrow$ DGluR-IIA animals (Mhc-Gal4 $\rightarrow$ DGluR-IIA vs Mhc-Gal4 $\rightarrow$ DGluR-IIA + IIB, $p \ll 0.005)$. Together with recent physiological examinations of DGluR-IIB overexpression (DiAntonio et al., 1999), these observations support the idea that the glutamate receptor subunits DGluR-IIA and DGluR-IIB serve complementary functions in the physiological and morphological development of larval NMJs. But how could such a counterbalancing role of DGluR-IIB relative to DGluR-IIA be mediated during the development of larval NMJs?

A possible answer to this question came from the striking observation that the knock-out of $\operatorname{dglur}-I I B$, which harbored only one genomic copy of dglur-IIA [dglur-II $A^{A 22} / d f(2 L) c l h 4 ; P(U A S$ gdglur-IIA $\left.)^{\mathrm{g} 10} /+\right]$, showed a strongly enhanced synaptic DGluRIIA immunoreactivity compared with wild-type controls (241 \pm $21 \% ; n=13 ; p<0.001)$, suggesting that DGluR-IIB expression might negatively influence the synaptic accumulation of DGluRIIA. Consistent with this idea, we found that the simultaneous transgenic overexpression of DGluR-IIA and DGluR-IIB did not result in an increased synaptic accumulation of DGluR-IIA subunits (DGluR-IIA immunoreactivity, $108 \pm 9 \%$ of control; $n=$ 14). Both of these genetic treatments resulted in junctional phenotypes that were fully consistent with our above-proposed role of altered DGluR-IIA expression levels: NMJs with unaltered synaptic DGluR-IIA levels showed a wild-type number of junctional boutons (Fig. 3D, Mhc-Gal4 $\rightarrow$ DGluR-IIA+IIB), whereas the above $\operatorname{dglur}-I I B$ knock-out animals displayed high levels of synaptic DGluR-IIA expression, significantly larger NMJs (Fig. $4 D$, right two bars) and a strongly enhanced junctional signal transmission (wild-type quantal sizes, larger evoked responses; Petersen et al., 1997). These data suggest that the junctional phenotypes that can be observed in animals with altered DGluR- 

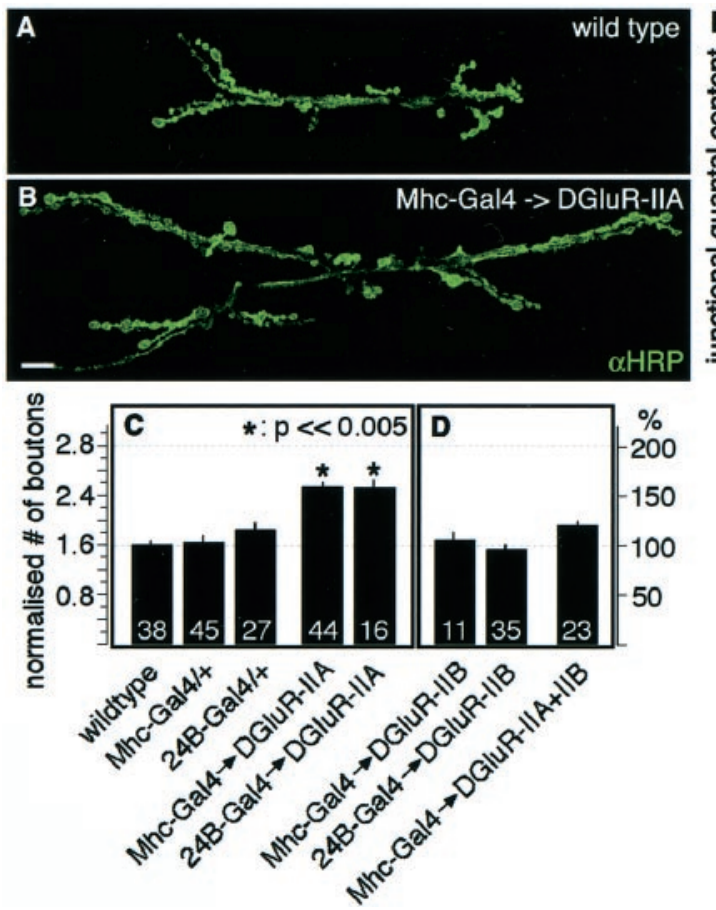

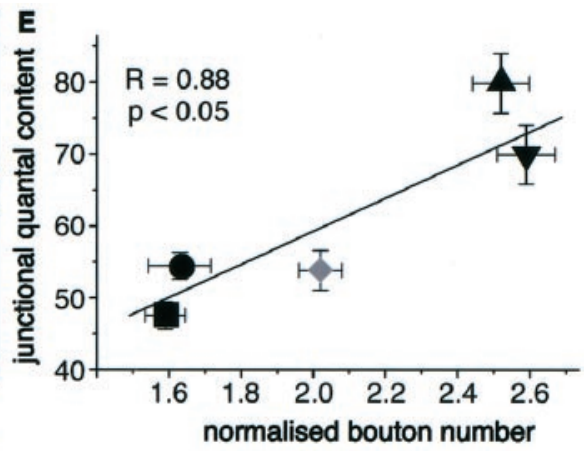

wild type

Mhc-Gal4/+

A Mhc-Gal4 $\rightarrow$ DGluR-IIA

> $p a b p^{-200 /+}$

pabp $p^{\infty 200}$, dglurllA $A^{\infty} /+$ without detectable effect on the morphological development of NMJs. Data are given as means \pm SEM. Scale bar, 50 $\mu \mathrm{m}$. E, Quantal content is plotted as a function of junction size. Both parameters (represented as means \pm SEM) correlate significantly among all analyzed genotypes.

IIB expression are mediated by a complementary regulation of the synaptic DGluR-IIA accumulation, which as shown above appears to be a quite potent mechanism to modulate both the strength of junctional signal transmission and endplate size.

The high sensitivity of developing larval NMJs toward the relative expression levels of DGluR-IIA and DGluR-IIB was further illustrated by the finding that animals that lost only one dglur-II A gene copy developed wild-type NMJ sizes (Fig. 4D, gray bar), whereas larvae that lost one copy of both genes, dglur-IIA and $d g l u r-I I B$, showed a small but significant increase in size of NMJs (Fig. 4D, hatched gray bar). Together, these results indicate that the regulated synaptic accumulation of DGluR-IIA is primarily responsible for the observed physiological and morphological strengthening of NMJs and that DGluR-IIB may be involved in the regulation of synaptic DGluR-IIA accumulation. However, because of the current lack of antibodies that recognize endogenous DGluR-IIB subunits we have not been able to analyze whether this subunit is indeed subject to a similar dynamic regulation as described for DGluR-IIA (Sigrist et al., 2000).

\section{Activity-induced morphological plasticity requires unrestricted DGluR-IIA expression}

To further elaborate the role of regulated DGluR-IIA and DGluR-IIB expression during activity-induced plasticity of larval NMJs, we genetically modified the relative expression of both subunits in three previously described plasticity mutants: an allele of $p a b p$ that resulted in an elevated subsynaptic appearance of large eIF4e-aggregates ( $p a b p^{P 970} /+$; Sigrist et al., 2000), a mutant with chronically enhanced neuronal activity levels (eag ${ }^{1}$, $\left.S h^{102} / Y\right)$, and a mutant with increased cellular cAMP-levels (dnc $c^{M 14} / Y$; Budnik et al., 1990; Schuster et al., 1996b). These three genotypes have been shown previously (Budnik et al., 1990; Schuster et al., 1996b; Sigrist et al., 2000) to develop significantly larger NMJs than wild-type controls (Fig. 4E,F, black bars) $(p \ll$ $0.001)$. The increase in junctional growth was partially suppressed by removing only one copy of the dglur-IIA gene (Fig. 4E,F, gray bars) most likely because of restricted synaptic expression of
DGluR-IIA throughout development. Consistent with our above results in otherwise wild-type backgrounds (Fig. 4D), the additional elimination of both dglur-IIB genes overcompensated for the loss of one dglur-IIA gene copy and overruled this growth suppression, resulting in the development of very large NMJs (Fig. $4 E$, right bar vs gray bars). In contrast, the additional junctional growth of $p a b p^{P 970} /+$ animals was completely abolished in dglur-IIA knock-out animals (Fig. 4E, hatched white bar), although this genotype harbored only one dglur-IIB gene copy. These data demonstrate that the enhanced bouton addition seen in various model genotypes of junctional plasticity requires increased synaptic DGluR-IIA expression. They further establish that changes at developing larval NMJs, which are induced by persistent hyperactivity, are mediated by an elevated synaptic accumulation of the glutamate receptor subunit DGluR-IIA.

\section{DGluR-IIA mediates junctional strengthening downstream of subsynaptic translation}

All above-described phenotypes that were caused by genetically increasing the DGluR-IIA expression were indistinguishable from those observed in animals with elevated subsynaptic translation. This similarity could be explained by a model in which one of the primary goals of subsynaptic translation is the local regulation of synaptic DGluR-IIA accumulation, which in turn mediates the physiological and morphological alterations seen. It is, however, similarly possible that increased synaptic DGluR-IIA expression results in locally enhanced signal transmission, which would further stimulate subsynaptic translation. This feedback could result in the local synthesis of molecular signals that organize for example synaptogenesis and morphological growth.

To differentiate between these possibilities, we assessed whether altered DGluR-IIA expression affects the appearance of subsynaptic translation aggregates. For this purpose, we quantified the number of boutons that showed subsynaptic immunoreactivity for the rate-limiting translation initiation factor eIF4E (Fig. 5A) in various genotypes. We found that the transgenic overexpression of DGluR-IIA in otherwise wild-type animals 

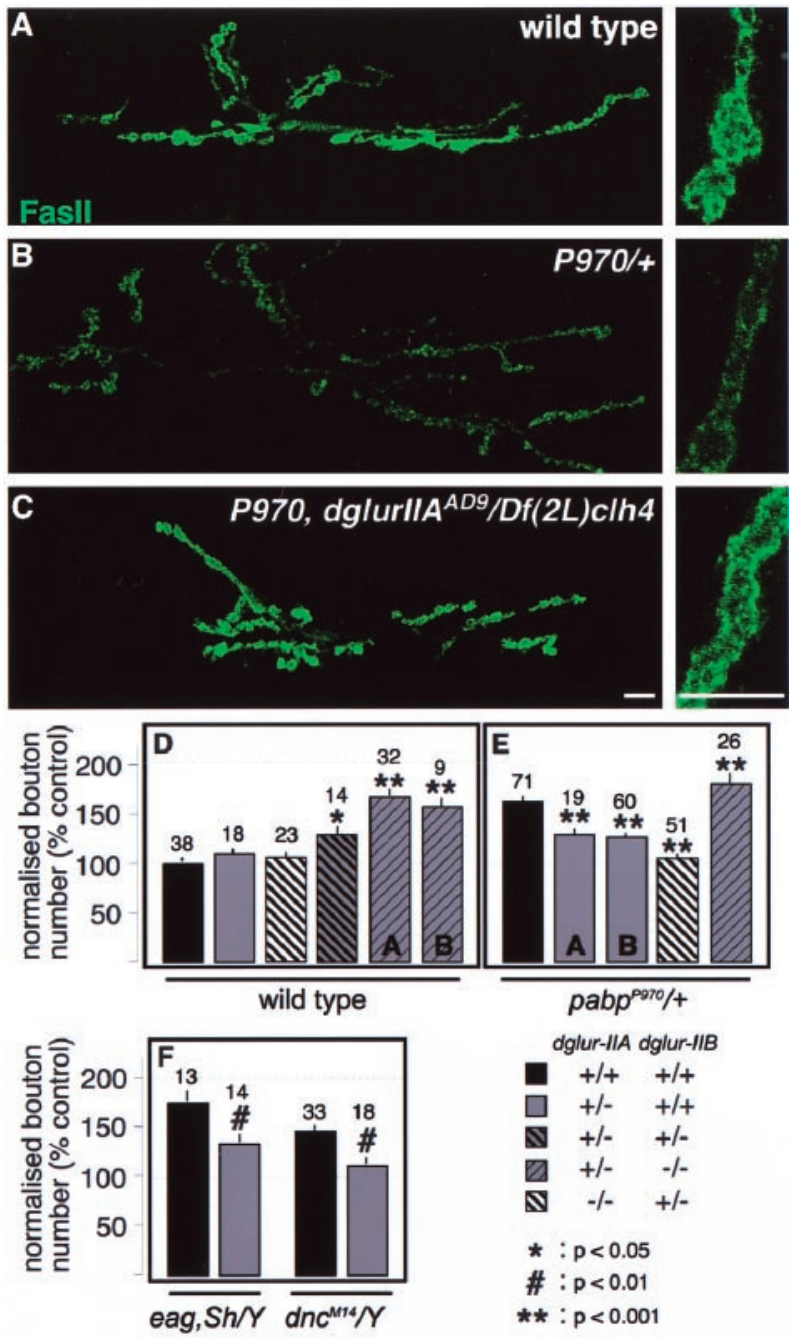

Figure 4. DGluR-IIA expression is necessary to allow activitydependent outgrowth of NMJs. $A-C$, Confocal images of FasII-labeled NMJs (muscle 6/7, A2) of the indicated genotypes as overviews (left panels) and in detail (right panels). Compared with wild-type controls, NMJs of $p a b p^{P 970} /+$ larvae show significantly more boutons $(E)$ and reduced perisynaptic FasII expression $(B)$. Both phenotypes are suppressed in animals that lost both copies of the $\operatorname{dglur}-I I A$ gene $\left[E ; p a b p^{P 970}\right.$,

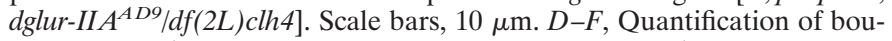
ton numbers (muscle 6/7, A2; see Materials and Methods) of the indicated genotypes (the genetic status of the $\operatorname{dglur}-I I A$ gene is symbolized by the bar color, that of the dglur-IIB gene by hatching; inset). $D$, In wild-type animals, the removal of one or both $\operatorname{dglur}-I I A$ gene copies does not affect the morphological development of NMJs. Loss of one or both dglur-IIB gene copies results in a stepwise and significant increase in junctional outgrowth. Genotypes: black bar, wild type; gray bar, dglur-II $A^{A D 9} /+$; hatched white bar, dglur-II $A^{A D 9} / \mathrm{df}(2 \mathrm{~L}) \mathrm{clh} 4$; hatched gray bar, dglur$I I A^{A 22} /+$; hatched gray bar $A$, dglur-II $A^{A 22} / \mathrm{df}(2 \mathrm{~L}) \mathrm{clh} 4$; P(UAS-gdglur$I I A)^{\mathrm{g} 10} /+$; hatched gray bar $B$, dglur-II $A^{A 22}$, P(UAS-gdglur-II $\left.A\right)^{\mathrm{g} 9} /$ $d f(2 L) c l h 4$. E, F, Increased junctional growth (black bar) seen in animals with elevated subsynaptic translation $\left(\mathrm{pabp}^{P 970} /+\right)$, increased neuronal activity $\left(e a g^{1}, S h^{102}\right)$, and cellular cAMP levels $\left(\right.$ dunce $\left.{ }^{M 14}\right)$ is strongly suppressed by the loss of one (gray bars) or both (hatched white bar) $\operatorname{dglur}-I I A$ gene copies. This suppression is overcome by the simultaneous loss of one gene copy of both receptor subunits ( $E$, right bar). Genotypes in $E$ : black bar, pabp ${ }^{P 970} /+$; bar $A$, pabp ${ }^{P 970} /$ dglur-II $A^{A D 9}$; bar B, pabp ${ }^{P 970}$, dglur-II $A^{A D 9} /+$; hatched white bar, pabp ${ }^{P 970}$, dglur-II $A^{A D 9} / d f(2 L)$ clh4; right bar, pabp ${ }^{P 970} /$ dglur-II $A^{A 22}$. Genotypes in $F$ : bar, eag ${ }^{I}, S h^{102} / Y$; left gray bar, eag ${ }^{1}, S^{102} / Y$; dglur-II $A^{A D^{9}} /+$; right black bar, dnc ${ }^{M 14} / Y$; right gray bar, $d n c^{M 14} / Y$; dglur-II $A^{A D 9} /+$. Data are represented as means \pm SEM; numbers of analyzed segments are given above bars.
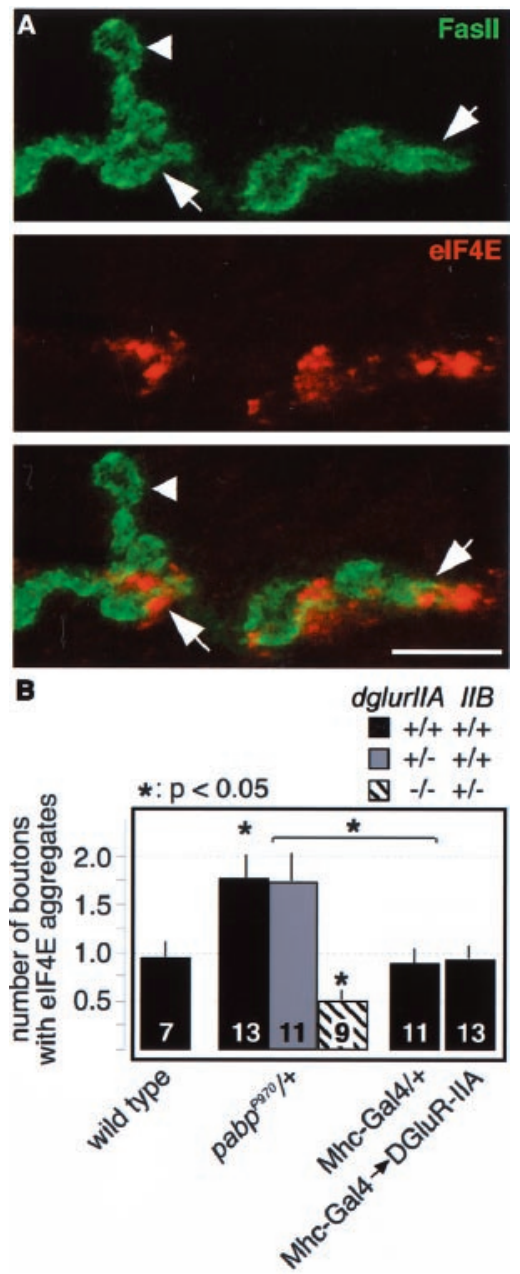

Figure 5. Altered DGluR-IIA expression does not affect subsynaptic translation. A, Confocal images of boutons labeled for FasII (green) and the translation initiation factor eIF4E (red). Marked are individual synaptic boutons that are positive (arrow) or negative (arrowhead) for subsynaptic translational aggregates. $B$, Quantification of subsynaptic translation aggregates in animals of the indicated genotypes (see Materials and Methods). The number of boutons with subsynaptic translation aggregates is significantly increased in $\mathrm{pabp}^{P 970} /+$ animals. Both transgenic overexpression of DGluR-IIA and loss of one dglur-IIA gene copy do not affect the levels of subsynaptic translation. Complete elimination of dglur$I I A$ in translation-sensitized animals $\left[\mathrm{pabp}^{P 970}\right.$ dglur-II $\left.A^{A D 9} / d f(2 L) \operatorname{clh} 4\right]$ results, however, in a strong reduction of subsynaptic translation, presumably attributable to the strong defects in synaptic signal transmission (Petersen et al., 1997). Data are plotted as means \pm SEM; numbers of analyzed animals are within bars. Scale bar, $10 \mu \mathrm{m}$.

(Mhc-Gal4 $\rightarrow$ DGluR-IIA) did not increase the appearance of large subsynaptic eIF4E aggregates compared with controls (Fig. $5 B$, Mhc-Gal4/+, wild type), suggesting that the DGluR-IIAinduced phenotypes are not mediated by a feedback enhancement of subsynaptic protein synthesis. Consistent with this observation, we did not find an alteration of subsynaptic translation levels in animals that lost one dglur-IIA gene copy in the translationally sensitized background pabp $^{P 970} /+\left(p^{9} a p^{P 970}\right.$, dglur$\left.I I A^{A D 9} /+\right)$ (Fig. 5B, gray bar). This result again demonstrates that the physiological and morphological phenotypes seen in this mutant (Figs. 2, 3E, 4E) depend on the expression level of DGluR-IIA, whose efficient upregulation appears here to be limited and thus restricts the implementation of protein synthesisdependent plasticity at larval NMJs. Therefore, our results show 
that subsynaptic translation mediates most of its effects by regulating the postsynaptic accumulation of DGluR-IIA, which in turn is responsible for the observed long-term strengthening.

Complete genetic elimination of dglur-IIA (dglur-II $\left.A^{-} / d f\right)$ was associated with a strong reduction of subsynaptic translation aggregates even in translationally sensitized $\mathrm{pabp}^{P 970} /+$ mutants (Fig. $5 B$, white bar). Because this mutant shows as a primary defect a considerably reduced postsynaptic sensitivity to released neurotransmitter (Petersen et al., 1997; DiAntonio et al., 1999), this result indicates that normal postsynaptic function is required to efficiently induce the formation of large subsynaptic aggregates of eIF4E.

\section{DGluR-IIA mediates structural outgrowth via the downregulation of perisynaptic Fasciclin II}

We have shown previously that genetically induced long-term strengthening of signal transmission and the associated increased junctional growth is accompanied by a small but significant reduction in the perisynaptic expression level of the cell adhesion molecule FasII (Grenningloh et al., 1991; Schuster et al., 1996b; Sigrist et al., 2000). This FasII downregulation has in fact been shown to be required for the morphological changes seen in the plasticity mutants $d n c^{M 14} / Y$ and $e^{2 a g}{ }^{1}, S h^{102} / Y$ (Schuster et al., 1996b). Intriguingly, we observed in this study a similar reduction of perisynaptic FasII levels in DGluR-IIA-overexpressing larvae (Fig. 1B, green channel), suggesting that an increased synaptic DGluR-IIA accumulation can elicit a specific perisynaptic downregulation of FasII to result in the observed morphological growth (Fig. 3). In addition, the FasII downregulation that could be seen in $p a b p^{P 970} /+$ animals (Fig. $4 B$ ) was suppressed in larvae with eliminated $\operatorname{dglur}$-IIA gene copies (Fig. $4 C$ ) and thus resulted in a suppressed junctional outgrowth (Fig. $4 E$, hatched white bar). This finding further supports the idea that a dynamic DGluR-IIA regulation is required to trigger FasII-mediated morphological changes at larval NMJs.

To experimentally test this hypothesis, we attempted to compete with the induced perisynaptic FasII downregulation by mildly overexpressing FasII specifically in a subset of motoneurons using a previously described combination of transgenes (E62-Gal4/UAS-FasII; Schuster et al., 1996b). However, because of the fact that the simultaneous overexpression of DGluR-IIA and FasII in different cells is impossible with the Gal4/UAS system, we performed this competition experiment in $\mathrm{pabp}^{P 970} /+$ animals, which as established above mediate their junctional effects via DGluR-IIA regulation. Consistent with the previously demonstrated role of FasII for junctional growth (Schuster et al., 1996b), we found that the additional presynaptic FasII expression was sufficient to suppress the additional outgrowth seen at $p_{a b p} p^{P 970} /+$ NMJs $\left(p^{2} a p^{P 970} /+, 100 \pm 5.4 \% ; n=12 ; p^{2} a p^{P 970}\right.$, E62-Gal4/UAS-FasII, $62.3 \pm 5.4 \% ; n=17 ; p \ll 0.001)$. We therefore conclude that activity-, translation-, and DGluR-IIAinduced morphological changes are mediated by an associated perisynaptic downregulation of the cell adhesion molecule FasII.

\section{Elevated DGluR-IIA expression increases the total number of active zones, but their spacing remains unaltered}

Strengthened signal transmission at NMJs as a whole may originate either from an elevated presynaptic vesicle release at preexisting synapses or from an increase in the total number of functional synapses. In support of the latter possibility, we have already found that the apparent number of DGluR-IIAexpressing synapses was obviously elevated (Fig. $1 B$ ) and that the frequency of spontaneous mEJCs was significantly increased at DGluR-IIA-overexpressing NMJs (Fig. 2C, white bars). These phenotypes were similar to those seen in larvae with elevated subsynaptic translation (Fig. 2B,C, $p a b p^{P 970} /+$ ), indicating that enhanced signal transmission at NMJs may be attributed to a DGluR-IIA mediated recruitment of functional release sites.

To assess whether DGluR-IIA upregulation is indeed associated with morphological alterations on the synaptic level, we reconstructed randomly chosen boutons from several type $\mathrm{Ib}$ axon branches per genotype from serially cut ultrathin sections. The following parameters were scored (Table 1): the total surface area of the analyzed boutons, the number of synapses and their sizes (reconstructed electron-dense membrane areas) (Fig. 6 $\mathrm{A}$, arrowheads), and the presence or absence of presynaptic T-shaped dense bodies ("T-bars") (Fig. 6B, arrows). Presynaptic T-bars are thought to label synapses with increasingly higher vesicle release probabilities and are therefore termed active zones (Cooper et al., 1995, 1996), whereas T-bar-free synapses lack the characteristic clusters of docked synaptic vesicles and are believed to represent sites of low vesicle release probability (Atwood et al., 1993; Wojtowicz et al., 1994; Atwood and Wojtowicz, 1999). From these raw data, we determined the density of T-bar-harboring and T-bar-free synapses on the bouton surface (number of synapses per surface area).

Our ultrastructural analysis revealed that the density of T-barcontaining synapses (Fig. 6C, gray bars) was unaltered in all reconstructed axonal branches of all examined genotypes (total of 23 branches from 10 NMJs, eight animals; Table 1). This included those genotypes that overexpressed DGluR-IIA directly (MhcGal4 $\rightarrow$ DGluR-IIA) or indirectly as a consequence of increased subsynaptic translation (Mhc-Gal4 $\rightarrow$ PABP and pabp EP0310/ $d f(2 R) P c l 7 b$; see Sigrist et al., 2000). It is therefore likely that the density of active zones is maintained in a homeostatic manner throughout an entire NMJ, suggesting that the total number of T-bar-harboring synapses is proportional to the size of the NMJ. We therefore conclude that the total number of T-bar-containing synapses (including those with more than one T-bar) is increased at NMJs with elevated synaptic levels of DGluR-IIA (Fig. 6C, black bars; Table 1).

It is interesting to note that this increase in the total number of active zones was associated with a considerable reduction in the density of synapses without T-bars (Fig. 6C, white bars; Table 1). A similar finding has been reported previously in other genotypes with increased junctional output (Stewart et al., 1996). These observations suggest that the presence of T-bars at synapses is not statically defined for example at a certain time point during synaptogenesis. Rather, T-bars appear to be recruited in a dynamic manner to previously T-bar-free synapses, resulting in a considerable alteration of the ratio between T-bar-containing active zones and T-bar-free synapses (Table 1).

These ultrastructural results have several implications. First, the increase in the total number of active zones (141-163\%; Table 1) can presynaptically account for the strengthening of junctional signal transmission seen in the above genotypes $(\sim 150 \%)$ (Fig. 2). Second, the increase in the number of presynaptic T-bars was induced solely by the postsynaptic overexpression of DGluR-IIA, suggesting that retrogradely acting signals control the number of active zones at NMJs (Petersen et al., 1997; Davis et al., 1998). Third, the here-observed homeostatic regulation of the active zone density is consistent with results from a previous study performed at NMJs of Drosophila and Sarcophaga (Meinertzhagen et al., 1998), suggesting that active synapses require for their 

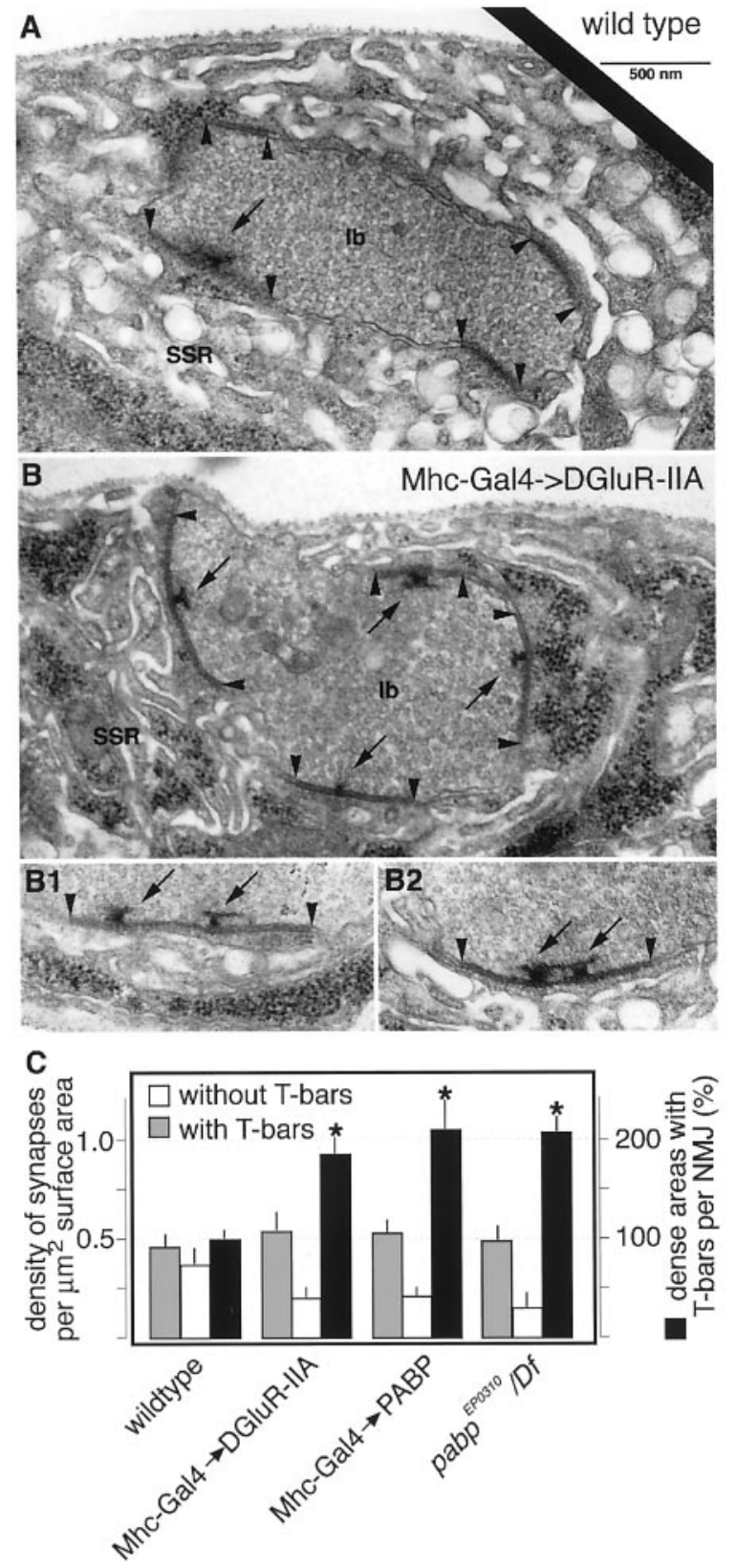

Figure 6. Ultrastructural effects of elevated DGluR-IIA expression. $A, B$, Representative transmission electron microscopic images of type Ib boutons (muscle 6/7, abdominal segment A2) in wild-type $(A)$ and DGluRIIA-overexpressing $(B)$ animals. Marked are synapses (dense areas between arrowheads), the subsynaptic reticulum (SSR), presynaptic dense bodies (T-bars; arrows), and complex synapses harboring two T-bar structures within a single electron dense area $(B 1, B 2)$. Large sequential series of such images were used to reconstruct junctional branches and to analyze ultrastructural changes (Table 1; Materials and Methods). $C$, Genotypes with transgenic overexpression of DGluR-IIA $($ Mhc-Gal4 $\rightarrow$ DGluR-IIA $)$ or animals with sensitized subsynaptic translation and concomitantly elevated DGluR-IIA expression [MhcGal4 $\rightarrow P A B P$, pabp $^{E P 0310} / d f(2 R) P c l 7 b$; Sigrist et al., 2000] show an unaltered density of synapses with T-bars (i.e., active zones; gray bars) and a reduced density of synapses without T-bars (white bars) compared with wild-type controls. This results in a significant increase in the total number of active zones per NMJ in animals with elevated DGluR-IIA expression (black bars; $p<0.01$ ). Data are taken from Table 1 and are given as means \pm SEM. function a typical perisynaptic area. It is therefore tempting to speculate that an increased functional recruitment of synapses may transiently result in an increased density of active zones, which may eventually trigger the additional outgrowth of boutons to restore a functionally preferred density of active zones. Such a combination of mechanisms may explain why NMJs with an increased strength of junctional signal transmission grow proportionally more boutons.

\section{DISCUSSION}

\section{DGluR-IIA expression controls the functional recruitment of active zones}

The focus of this study was the question of how local subsynaptic protein synthesis can regulate the transmission strength and the morphological development of larval neuromuscular junctions of Drosophila (Sigrist et al., 2000). Our results show that the increased synaptic accumulation of the glutamate receptor subunit DGluR-IIA alone is responsible for the functional recruitment of additional synapses within a given NMJ. This was evident in an apparent increase in the number of DGluR-IIA-expressing postsynapses on transgenic overexpression of DGluR-IIA (Fig. 1 ), an increased total number of T-bar-harboring release sites per NMJ (Fig. 6), an increased frequency of spontaneous vesicle fusion events (Fig. 2C), and significantly larger junctional responses on nerve stimulation (eEJCs) (Fig. 2) compared with control animals. These physiological changes, which have been evoked solely by manipulating the expression level of DGluRIIA, were indistinguishable from those seen in animals with genetically increased subsynaptic translation, and they were suppressed in the latter genotypes by reducing the dglur-IIA gene doses (Figs. 2-4). These observations suggest that most if not all of the physiological effects of subsynaptic protein synthesis at NMJs are mediated by increased synaptic expression of the glutamate receptor subunit DGluR-IIA. Because subsynaptically stored mRNAs encoding DGluR-IIA represent a likely substrate of synaptic translation (Sigrist et al., 2000), the local synthesis of DGluR-IIA subunits and their subsequent synaptic delivery could therefore provide the means for a site-specific functional recruitment of synapses and thus for local alterations of glutamatergic signal transmission.

Interestingly, we found that the synaptic expression level of DGluR-IIA and its associated physiological phenotypes are inversely related to the expression of the glutamate receptor subunit DGluR-IIB (Petersen et al., 1997): a reduced dglur-IIB gene copy number resulted in a significant increase of synaptic DGluRIIA accumulation, whereas the transgenic overexpression of DGluR-IIB reduced synaptic DGluR-IIA levels. One possibility to explain this inverse relationship of both glutamate receptor expression levels could be a competition of both subunits in the formation of hetero-oligomeric receptor complexes. Another possibility may reside in the previously described opposing roles of DGluR-IIA and DGluR-IIB for synaptic signal transmission (DiAntonio et al., 1999): synapses expressing DGluR-IIA resemble wild-type transmission characteristics, whereas DGluR-IIBexpressing synapses exhibit very fast desensitization kinetics, resulting in strongly reduced quantal sizes. Given that NMJs with small quantal sizes were accompanied by suppressed subsynaptic protein synthesis (Fig. 5B, hatched bar) this could result in an inefficient subsynaptic synthesis and a reduced synaptic delivery of DGluR-IIA in DGluR-IIB-overexpressing animals. In turn, synapses with reduced or no DGluR-IIB may efficiently activate the DGluR-IIA synthesis and their synaptic deposition. Although 
Table 1. Summary of ultrastructural analysis

\begin{tabular}{|c|c|c|c|c|}
\hline \multirow[b]{2}{*}{ Characteristic } & \multicolumn{4}{|l|}{ Genotype } \\
\hline & $+/+$ & $\begin{array}{l}\text { Mhc-Gal4 } \rightarrow \\
\text { DGluRIIA }\end{array}$ & $\begin{array}{l}\text { Mhc-Gal4 } \rightarrow \\
\text { PABP }\end{array}$ & $\mathrm{pabp}^{E P 0310} / \mathrm{df}$ \\
\hline Number of branches analyzed & 4 & 4 & 10 & 5 \\
\hline Total length of branches sectioned $(\mu \mathrm{m})$ & 37.8 & 39.4 & 83.8 & 41.1 \\
\hline Total junction surface sectioned $\left(\mu \mathrm{m}^{2}\right)$ & 174.0 & 241.1 & 368.1 & 228.2 \\
\hline Total number dense areas observed & 144 & 178 & 268 & 141 \\
\hline Number dense areas with one T-bar & 70 & 108 & 158 & 99 \\
\hline Number of dense areas with more than one T-bar & 9 & 17 & 33 & 10 \\
\hline Number dense areas without T-bar & 65 & 58 & 77 & 32 \\
\hline Mean size dense areas $\left(\mu \mathrm{m}^{2}\right)$ & $0.285 \pm 0.015$ & $0.329 \pm 0.018$ & $0.225 \pm 0.010$ & $0.287 \pm 0.015$ \\
\hline Dense areas/surface area $\left(\mu \mathrm{m}^{2}\right)$ & $0.83 \pm 0.08$ & $0.74 \pm 0.09$ & $0.74 \pm 0.08$ & $0.64 \pm 0.06$ \\
\hline Dense areas with T-bars/surface area $\left(\mu \mathrm{m}^{2}\right)$ & $0.46 \pm 0.06$ & $0.54 \pm 0.09$ & $0.53 \pm 0.06$ & $0.49 \pm 0.07$ \\
\hline Dense areas without T-bar/surface area $\left(\mu \mathrm{m}^{2}\right)$ & $0.37 \pm 0.08$ & $0.20 \pm 0.05$ & $0.21 \pm 0.04$ & $0.15 \pm 0.07$ \\
\hline Size of entire NMJ $(\%)$ & $100 \pm 4$ & $158 \pm 5$ & $183 \pm 13$ & $196 \pm 6$ \\
\hline (number of boutons/muscle surface area) & $(1.59 \pm 0.06)$ & $(2.52 \pm 0.08)$ & $(2.91 \pm 0.20)$ & $(3.11 \pm 0.09)$ \\
\hline Total number dense areas per entire NMJ $(\%)$ & $\begin{array}{c}100 \pm 8 \\
(1.38 \pm 0.10)\end{array}$ & $\begin{array}{c}141 \pm 9 \\
(1.86 \pm 0.18)\end{array}$ & $\begin{array}{c}163 \pm 17 \\
(8.15 \pm 0.22)\end{array}$ & $\begin{array}{c}151 \pm 8 \\
(1.99 \pm 0.11)\end{array}$ \\
\hline Dense areas with T-bars per entire NMJ (\%) & $\begin{array}{c}100 \pm 11 \\
(0.70 \pm 0.08)\end{array}$ & $\begin{array}{c}188 \pm 18 \\
(1.36 \pm 0.12)\end{array}$ & $\begin{array}{c}211 \pm 29 \\
(1.54 \pm 0.21)\end{array}$ & $\begin{array}{c}208 \pm 15 \\
(1.52 \pm 0.11)\end{array}$ \\
\hline Dense areas without T-bar per entire NMJ $(\%)$ & $\begin{array}{c}100 \pm 17 \\
(0.59 \pm 0.10)\end{array}$ & $\begin{array}{c}85 \pm 15 \\
(0.50 \pm 0.09)\end{array}$ & $\begin{array}{c}103 \pm 34 \\
(0.61 \pm 0.20)\end{array}$ & $\begin{array}{c}80 \pm 19 \\
(0.47 \pm 0.11)\end{array}$ \\
\hline Active zones $(\%)$ & 56 & 72 & 73 & 76 \\
\hline
\end{tabular}

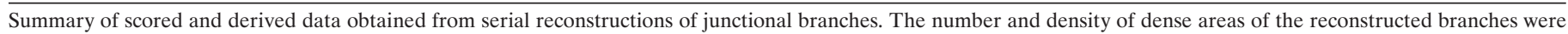

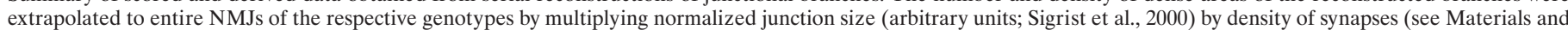

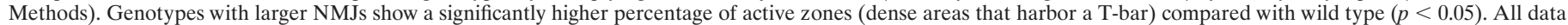
were obtained from individual branches and then combined to mean values \pm SEM per genotype.

we are currently unable to differentiate between these and other possibilities, it is important to note that NMJs are apparently equipped with two subunit-specific mechanisms, which because of their opposing effects on synaptic DGluR-IIA accumulation are well suited to tightly control the subunit composition of postsynaptic glutamate receptors.

On the basis of these data, it appears that a crucial factor for the implementation of persistently strengthened junctional signal transmission is the controlled upregulation of DGluR-IIA, which results in the functional recruitment of additional synapses. These added synapses showed postsynaptic responses to released quanta of glutamate that were typical for wild-type NMJs (Fig. 2), suggesting that increased DGluR-IIA expression results primarily in a larger number of normally operating postsynapses. Very strong overexpression of DGluR-IIA, which has been achieved previously using a cDNA-based transgene, appears to further increase the amount of DGluR-IIA at individual postsynapses and has been shown to result in a dose-dependent increase of miniature excitatory junctional potential (mEJP) amplitudes (Petersen et al., 1997; DiAntonio et al., 1999). These findings suggest that not only the number of responsive postsynapses can be changed by DGluR-IIA, but also the postsynaptic sensitivity can be changed. They further support the idea that the number of postsynaptic glutamate receptor complexes per synapse determines the amplitudes of mEJPs (DiAntonio et al., 1999), and they are consistent with results from hippocampal synapses, which propose that a neurotransmitter from a single vesicle saturates all postsynaptic glutamate receptors of that synapse (Tang et al., 1994).

Strengthening of glutamatergic synapses and activation of silent postsynapses during long-term potentiation has recently gained much attention (Malinow et al., 2000). Several lines of evidence have suggested that the targeted trafficking of specific glutamate receptor subunits and their incorporation into preexisting synapses represent a prominent route of synaptic activation and functional modification in hippocampal preparations (Jia et al., 1996; Nayak et al., 1998; Shi et al., 1999, 2001; Hayashi et al., 2000; Kacharmina et al., 2000; Zhu et al., 2000). Our results from the Drosophila NMJ indicate that these glutamatergic synapses use similar postsynaptic mechanisms to functionally recruit additional synapses, indicating that the local synthesis and the targeted trafficking of receptor subunits may represent an evolutionary conserved mode to alter glutamatergic circuits in a sitespecific manner.

\section{Does the number of active zones control the number of boutons?}

There is increasing evidence from this and several other recent studies (Cheung et al., 1999; Sigrist et al., 2000; Sanyal et al., 2002) that the strength of junctional signal transmission is correlated with the number of synapse-harboring boutons (Fig. 3D). Strikingly, we found in this study that the density of active zones, which represent sites of high-probability vesicle release (Wojtowicz et al., 1994), is approximately constant within individual boutons in all analyzed genotypes (Fig. 6; Table 1), even in NMJs with strongly enhanced signal transmission. This observation is consistent with a recent report (Meinertzhagen et al., 1998) suggesting that the spacing of active zones at NMJs and in the visual system of Drosophila and Sarcophaga is tightly regulated, presumably because each active zone requires a large enough surrounding surface area for proper function. It therefore seems likely that synapse recruitment leads to a transient increase in the 


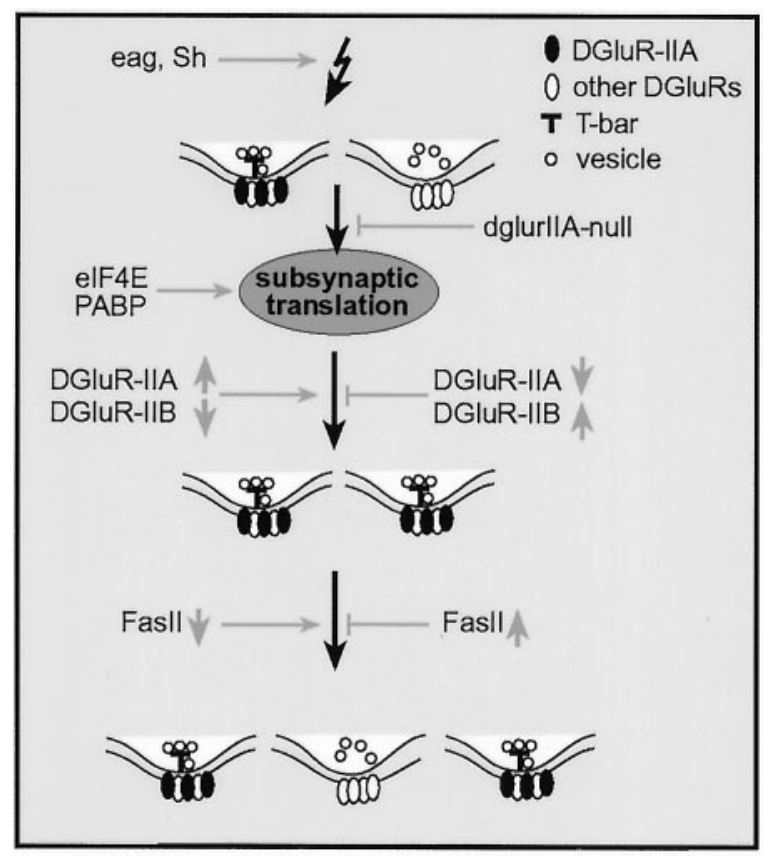

Figure 7. Model of activity-dependent long-term strengthening of signal transmission mediated by the regulated synaptic expression of DGluRIIA. Elevated motoneuron activity $\left(\mathrm{eag}^{1}, \mathrm{Sh}^{102}\right)$ leads to increased synaptic transmission triggering the formation of subsynaptic aggregates of specialized translation (Sigrist et al., 2000). This activity-dependent process is significantly suppressed in mutants with defective synaptic signal transmission (dglur-IIA-null); it is enhanced in animals with genetically sensitized subsynaptic translation (e.g., overexpression of eIF4E or PABP). Stimulated subsynaptic protein synthesis results in an increased synaptic accumulation of the glutamate receptor subunit DGluR-IIA, a process that appears to be counteracted by the subunit DGluR-IIB. Such an increased synaptic DGluR-IIA accumulation is associated via yet unknown retrograde signals with an increased functional recruitment of active zones (T-bar harboring synapses), which may result in a transiently increased density of active zones (e.g., active zones with more than one T-bar; Table 1). To restore the typical space requirements of active zones, the NMJ expands using a process that depends on the downregulation of the perisynaptically expressed cell adhesion molecule Fasciclin II.

density of active zones (Fig. 7) at larval NMJs of Drosophila, which are induced to grow to provide the now-required additional synaptic surface area. Interestingly, this growth does not involve a simple size increase of preexisting boutons (Figs. 3, 4), but it uses in a FasII-dependent manner (Fig. 4B) (Schuster et al., 1996) the rather costly addition of new boutons to NMJs. This suggests that the axonal compartmentalization, which is given in form of type I boutons, generates functional units that, similarly to the spacing of active zones, need to be homeostatically preserved. It therefore appears that the consistent correlation between the strength of junctional signal transmission and the number of junctional boutons (Fig. 3D) (Cheung et al., 1999; Sigrist et al., 2000; Sanyal et al., 2002) reflects the consolidation of induced functional changes, which include the functional recruitment of synapses and their distribution in newly grown boutons.

\section{Two modes of NMJ development: programmed development and activity-dependent plasticity}

On the basis of the prominent role of the glutamate receptor subunit DGluR-IIA during long-term strengthening of signal transmission, the question arises of whether NMJs can develop without DGluR-IIA. Surprisingly, NMJs with genetically eliminated DGluR-IIA expression developed to a size that resembled that of wild-type NMJs (Fig. 4D, white bar) despite strong defects in synaptic signal transmission (Petersen et al., 1997; DiAntonio et al., 1999). The same effect of eliminated DGluR-IIA expression was found in animals with increased subsynaptic protein synthesis (Fig. 4E, white bar), which normally develop significantly larger NMJs (Fig. 4E, black bar). Moreover, mutants in the synaptic vesicle protein synaptotagmin that have substantial defects in junctional signal transmission showed similar basal development of NMJs (DiAntonio and Schwarz, 1994). These observations demonstrate that neither DGluR-IIA expression itself nor intact synaptic physiology or subsynaptic translation (Fig. $5 B$, white bar) is required to develop NMJs with a relatively simple morphology. They suggest that larval NMJs can develop according to a program that appears to be independent of neuronal activity and the expression of the glutamate receptor subunit DGluR-IIA. This "programmed development" appears to establish a minimal innervation that would typically ensure baseline synaptic signal transmission and muscle contraction.

Superimposed on this programmed development, we describe some of the mechanisms underlying the functional and structural modulation of the initially established synaptic connectivity. Because postsynaptic DGluR-IIA expression plays a key role in this form of plasticity, which is likely regulated by neuronal activity and local subsynaptic protein synthesis (Sigrist et al., 2000), we propose that the "activity-dependent" mode of junctional development (Fig. 7) helps adjust the junctional performance to the prevailing needs of the individual animal. A similar concept of activity-induced modifications of previously established neural circuits has been implicated previously in the development and functional tuning of various neural networks (Goodman and Shatz, 1993; Katz and Shatz, 1996), for example, during the formation of barrels in the somatosensory cortex (O'Leary, 1994; Feldman et al., 1999; Erzurumlu and Kind, 2001), and of ocular dominance columns in the primary visual cortex (Kalil et al., 1986; Shatz and Stryker, 1988). On the basis of these similarities, the molecular and genetic analysis of developing NMJs of $\mathrm{Dro}$ sophila might yield further important insights into the mechanisms underlying the activity-dependent remodeling of synaptic networks.

\section{REFERENCES}

Aberle H, Haghighi AP, Fetter RD, McCabe BD, Magalhaes TR, Goodman CS (2002) Wishful thinking encodes a BMP type II receptor that regulates synaptic growth in Drosophila. Neuron 33:545-558.

Atwood HL, Wojtowicz JM (1999) Silent synapses in neural plasticity: current evidence. Learn Mem 6:542-571.

Atwood HL, Govind CK, Wu CF (1993) Differential ultrastructure of synaptic terminals on ventral longitudinal abdominal muscles in Drosophila larvae. J Neurobiol 24:1008-1024.

Budnik V, Zhong Y, Wu CF (1990) Morphological plasticity of motor axons in Drosophila mutants with altered excitability. J Neurosci 10:3754-3768.

Casadio A, Martin KC, Giustetto M, Zhu HX, Chen M, Bartsch D, Bailey CH, Kandel ER (1999) A transient, neuron-wide form of CREBmediated long-term facilitation can be stabilized at specific synapses by local protein synthesis. Cell 99:221-237.

Cheung US, Shayan AJ, Boulianne GL, Atwood HL (1999) Drosophila larval neuromuscular junctions responses to reduction of cAMP in the nervous system. J Neurobiol 40:1-13.

Cooper RL, Marin L, Atwood HL (1995) Synaptic differentiation of a single motor neuron: conjoint definition of transmitter release, presynaptic calcium signals, and ultrastructure. J Neurosci 15:4209-4222.

Cooper RL, Winslow JL, Govind CK, Atwood HL (1996) Synaptic structural complexity as a factor enhancing probability of calciummediated transmitter release. J Neurophysiol 75:2451-2466.

Davis GW, DiAntonio A, Petersen SA, Goodman CS (1998) Postsynaptic PKA controls quantal size and reveals a retrograde signal that regulates presynaptic transmitter release in Drosophila. Neuron 20:305-315. 
DiAntonio A, Schwarz TL (1994) The effect on synaptic physiology of synaptotagmin mutations in Drosophila. Neuron 12:909-920.

DiAntonio A, Petersen SA, Heckmann M, Goodman CS (1999) Glutamate receptor expression regulates quantal size and quantal content at the Drosophila neuromuscular junction. J Neurosci 19:3023-3032.

DiAntonio A, Haghighi AP, Portman SL, Lee JD, Amaranto AM, Goodman CS (2001) Ubiquitination-dependent mechanisms regulate synaptic growth and function. Nature 412:449-452.

Erzurumlu RS, Kind PS (2001) Neural activity: sculptor of "barrels" in the neocortex. Trends Neurosci 24:589-595.

Featherstone DE, Rushton EM, Hilderbrand-Chae M, Phillips M, Jackson FR, Broadie K (2000) Presynaptic glutamic acid decarboxylase is required for induction of the postsynaptic receptor field at a glutamatergic synapse. Neuron 27:71-84.

Feldman DE, Nicoll RA, Malenka RC (1999) Synaptic plasticity at thalamocortical synapses in developing rat somatosensory cortex: LTP, LTD and silent synapses. J Neurobiol 41:92-101.

Frey U, Morris RG (1997) Synaptic tagging and long-term potentiation. Nature 385:533-536.

Gallie DRA (1998) A tale of two termini: a functional interaction between the termini of a mRNA is a prerequisite for efficient translation initiation. Gene 216:1-11.

Goodman CS, Shatz CJ (1993) Developmental mechanisms that generate precise patterns of neuronal connectivity. Cell 72:77-98.

Grenningloh G, Rehm EJ, Goodman CS (1991) Genetic analysis of growth cone guidance in Drosophila Fasciclin II functions as a neuronal recognition molecule. Cell 67:45-58.

Hayashi Y, Shi S-H, Esteban JA, Piccini A, Poncer J-C, Malinow R (2000) Driving AMPA receptors into synapses by LTP and CaMKII: requirement for GluR1 and PDZ domain interaction. Science 287:2262-2267.

Huber KM, Kayser MS, Bear MF (2000) Role for rapid dendritic protein synthesis in hippocampal mGluR-dependent long-term depression. Science 288:1254-1256.

Jia Z, Agopyan N, Miu P, Xiong Z, Henderson J, Gerlai R, Taverna FA, Velumian A, MacDonald J, Carlen P, Abramownewerly W, Roder J (1996) Enhanced LTP in mice deficient in the AMPA receptor GluR2. Neuron 17:945-956.

Kacharmina JE, Job C, Crino P, Eberwine J (2000) Stimulation of glutamate receptor protein synthesis and membrane insertion within isolated neuronal dendrites. Proc Natl Acad Sci USA 97:11545-11550.

Kalil RE, Dubin MW, Scott G, Stark LA (1986) Elimination of action potentials blocks the structural development of retinogeniculate synapses. Nature 323:156-158.

Kang HJ, Schuman EM (1996) A requirement for local protein synthesis in neurotrophin-induced hippocampal synaptic plasticity. Science 273:1402-1406.

Katz LC, Shatz CJ (1996) Synaptic activity and the construction of cortical circuits. Science 274:1133-1138.

Linden DJ (1996) A protein synthesis-dependent late phase of cerebellar long-term depression. Neuron 17:483-490.

Malinow R, Mainen ZF, Hayashi Y (2000) LTP mechanisms: from silence to four-lane traffic. Curr Opin Neurobiol 10:352-357.

Manahan-Vaughan D, Kulla A, Frey JU (2000) Requirement of translation but not transcription for the maintenance of long-term depression in the CA1 region of freely moving rats. J Neurosci 20:8572-8576.

Martin KC, Casadio A, Zhu HX, Yaping EP, Rose JC, Chen M, Bailey $\mathrm{CH}$, Kandel ER (1997) Synapse-specific, long-form facilitation of Aplysia sensory to motor synapses-a function for local protein synthesis in memory storage. Cell 91:927-938.

Martin KC, Barad M, Kandel ER (2000) Local protein synthesis and its role in synapse-specific plasticity. Curr Opin Neurobiol 10:587-592.

Meinertzhagen IA, Govind CK, Stewart BA, Carter JM, Atwood HL (1998) Regulated spacing of synapses and presynaptic active zones at larval neuromuscular junctions in different genotypes of the flies Drosophila and Sarcophaga. J Comp Neurol 393:482-492.

Nayak A, Zastrow DJ, Lickteig R, Zahniser NR, Browning MD (1998)
Maintenance of late-phase LTP is accompanied by PKA-dependent increase in AMPA receptor synthesis. Nature 394:680-683.

O'Leary DDM (1994) Development critical period plasticity, and adult reorganizations of mammalian somatosensory systems. Curr Biol 4:535-544.

Paradis S, Sweeney ST, Davis GW (2001) Homeostatic control of presynaptic release is triggered by postsynaptic membrane depolarization. Neuron 30:737-749.

Parnas P, Haghighi AP, Fetter RD, Kim SW, Goodman CS (2001) Regulation of postsynaptic structure and protein localization by the rho-type guanine nucleotide exchange factor dPix. Neuron 32:415-424.

Petersen SA, Fetter RD, Noordermeer JN, Goodman CS, DiAntonio A (1997) Genetic analysis of glutamate receptors in Drosophila reveals a retrograde signal regulating presynaptic transmitter release. Neuron 19:1237-1248.

Saitoe M, Tanaka S, Takata K, Kidokoro Y (1997) Neural activity affects distribution of glutamate receptors during neuromuscular junction formation in Drosophila embryos. Dev Biol 184:48-60.

Sanyal S, Sandstrom DJ, Hoeffer CA, Ramaswami M (2002) AP1 functions upstream of CREB to control synaptic plasticity in Drosophila. Nature 416:870-874.

Schneider N, Schwartz JM, Kohler J, Becker M, Schwarz H, Gerisch G (2000) Golvesin-GFP fusions as distinct markers for Golgi and postGolgi vesicles in Dictyostelium cells. Biol Cell 92:495-511.

Schuster CM, Ultsch A, Schloss P, Cox JA, Schmitt B, Betz H (1991) Molecular cloning of an invertebrate glutamate receptor subunit expressed in Drosophila muscle. Science 254:112-114.

Schuster CM, Davis GW, Fetter RD, Goodman CS (1996a) Genetic dissection of structural and functional components of synaptic plasticity I: Fasciclin II controls synaptic stabilization and growth. Neuron 17:641-654.

Schuster CM, Davis GW, Fetter RD, Goodman CS (1996b) Genetic dissection of structural and functional components of synaptic plasticity II: Fasciclin II controls presynaptic structural plasticity. Neuron 17:655-667.

Shatz CJ, Stryker MP (1988) Prenatal tetrodotoxin infusion blocks segregation of retinogeniculate afferents. Science 242:87-89.

Shi SH, Hayashi Y, Petralia RS, Zaman SH, Wenthold RJ, Svoboda K, Malinow R (1999) Rapid spine delivery and redistribution of AMPA receptors after synaptic NMDA receptor activation. Science 284:1811-1816.

Shi SH, Hayashi Y, Esteban JA, Malinow R (2001) Subunit-specific rules governing AMPA receptor trafficking to synapses in hippocampal pyramidal neurons. Cell 105:331-343.

Sigrist SJ, Thiel PR, Reiff DF, Lachance PE, Lasko P, Schuster CM (2000) Postsynaptic translation affects the efficacy and morphology of neuromuscular junctions. Nature 405:1062-1065.

Sone M, Suzuki E, Hoshino M, Hou D, Kuromi H, Fukata M, Kuroda S, Kaibuchi K, Nabeshima Y-I, Hama C (2000) Synaptic development is controlled in the periactive zones of Drosophila synapses. Development 127:4157-4168.

Stewart BA, Schuster CM, Goodman CS, Atwood HL (1996) Homeostasis of synaptic transmission in Drosophila with genetically altered nerve terminal morphology. J Neurosci 16:3877-3886.

Tang CM, Margulis M, Shi QY, Fielding A (1994) Saturation of postsynaptic glutamate receptors after quantal release of neurotransmitter. Neuron 13:1385-1393.

Wan HI, DiAntonio A, Fetter RD, Bergstrom K, Strauss R, Goodman CS (2000) Highwire regulates synaptic growth in Drosophila. Neuron 26:313-329.

Wojtowicz JM, Marin L, Atwood HL (1994) Activity-induced changes in synaptic release sites at the crayfish neuromuscular junction. J Neurosci 14:3688-3703.

Zhu JJ, Esteban JA, Hayashi Y, Malinow R (2000) Postnatal synaptic potentiation: delivery of GluR4-containing AMPA receptors by spontaneous activity. Nat Neurosci 3:1098-1106. 